\title{
2019 novel coronavirus pneumonia with onset of dizziness: a case report
}

\author{
Zhaohong Kong', Jingwei Wang ${ }^{2}$, Tao Li ${ }^{1}$, Zhaohui Zhang ${ }^{1}$, Jiang Jian ${ }^{1}$ \\ ${ }^{1}$ Department of Neurology, ${ }^{2}$ Department of Medical Molecular Laboratory, Renmin Hospital of Wuhan University, Wuhan 430060, China \\ Correspondence to: Zhaohui Zhang, MD, PhD. Department of Neurology, Renmin Hospital of Wuhan University, Wuhan 430060, China. \\ Email: zhzhqing1990@163.com; Jiang Jian, MD, PhD. Department of Neurology \& Infectious Disease, Renmin Hospital of Wuhan University, \\ Wuhan 430060, China. Email: jiangjian97@126.com.
}

\begin{abstract}
The 2019 novel coronavirus (2019-nCoV) epidemic continues, with the number of infections and deaths increasing. The respiratory tract is the main route of transmission of the virus, and the majority of symptoms are respiratory relative. Until now, there has been no reports concerning the nervous system onset. We present a 2019-nCoV patient with the onset of simple dizziness, accompanied by dry throat, no fever, no cough, no headache, no mental abnormality, and no obvious abnormality in the nuclear magnetic resonance imaging (MRI) of the head. Meanwhile, chest computed tomography (CT) scans showed multiple small spot shadows and interstitial changes in the early stage, especially in the extrapulmonary zone. There was a development of multiple ground-glass shadows and infiltrative shadows in both lungs with mild pleural effusion. The nucleic acid gene detection was positive, and thus the diagnosis of 2019-nCoV was confirmed. At last, the prognosis was good after active treatment. After antiviral and anti-infective treatment, the symptoms recovered. We presume that 2019-nCoV can also manifest in the nervous system alone, and lung CT, which has relative specificity, should be used as a routine screening method.
\end{abstract}

Keywords: Coronavirus pneumonia (CoV); dizziness; nervous system symptoms; diagnosis; case report

Submitted Feb 02, 2020. Accepted for publication Mar 04, 2020.

doi: $10.21037 /$ atm.2020.03.89

View this article at: http://dx.doi.org/10.21037/atm.2020.03.89

\section{Introduction}

In the first decade of the $21^{\text {st }}$ century, the number of coronaviruses (CoVs) has increased, and new cases are emerging (1). However, at present, most people's understanding of coronavirus comes from severe acute respiratory syndrome (SARS) $\mathrm{CoV}$ and Middle East respiratory syndrome (MERS) CoV. In December 2019, a group of patients In Wuhan, China, had pneumonia initially which was later found to be related to a seafood wholesale market (2). Researchers isolated a new coronavirus from the human airway epithelial cells here, which formed another branch of the sarbecev subgenus, the original coronavirus subfamily. On January 12, 2020, the virus was officially named the 2019 novel coronavirus pneumonia (2019-nCoV) by the World Health Organization (WHO) (3). 2019-nCoV is the seventh member of the coronavirus family to infect humans and is being monitored and investigated further. As of this writing, the 2019-nCoV epidemic continues, with the number of infections and deaths increasing.

Coronaviruses are enveloped RNA viruses that are commonly found in humans, other mammals, and birds, and can cause respiratory, intestinal, liver, and nervous system diseases $(4,5)$. Six coronaviruses are known to cause human diseases (6), and four viruses-229E, OC43, nl63, and hku1-are common and typically cause common cold symptoms in immunocompetent individuals (6). The other 2 viruses, severe acute SARS CoV and MERS $\mathrm{CoV}$, are caused by zoonotic infectious diseases (7), and can sometimes be fatal to those infected. The cause of the severe acute respiratory syndrome outbreak in 2003 was found to be in the Guangdong Province of China (8-10). MERS $\mathrm{CoV}$ is a particularly severe respiratory disease that broke out in the Middle East in 2012 (11). Given the 
prevalence and wide distribution of the coronavirus, its substantial genetic diversity, the frequent recombination of the genome, and the increasing contact between humans and animals, new coronaviruses may appear more regularly in humans due to cross-species infection and occasional spillover events $(7,12)$.

Coronavirus can cause respiratory, digestive, and nervous system diseases in humans and animals. The new coronavirus is a strain of coronavirus that has never been found in the human body before (3). 2019-nCoV, which has begun the epidemic of viral pneumonia in Wuhan, is quite different from SARS and MERS. Through the experience of our hospital in dealing with the 2019-nCoV outbreak in the past month and from reviewing the relevant literature, it can be concluded that most of the existing clinical data focuses on the initial respiratory symptoms, while there are no reports concerning 2019-nCoV onset related to dizziness alone. Given this lack, we here report the case diagnosed in our hospital in the hope of improving early recognition of the virus and controlling the disease in the further clinical experience. We present the following case in accordance with the CARE Guidelines (13).

\section{Case presentation}

A 53-year-old female who came to our department on December 26, 2019, due to "sudden dizziness for 3 days" accompanied by dry throat. There was no headache, tinnitus, fever, cough, headache, mental disorder, and numbness when getting up. Chest computed tomography (CT) showed normal results. There were no other accompanying symptoms. Her throat was dry and uncomfortable in the first half month of admission, and betahistine was used in the outside of hospital. And she was admitted to our department as "dizziness to be examined". She reported that her cephalosporin skin test was positive and that she was sleeping poorly. The current history of 5 items showed poor diet, normal physical strength, and no notable change in weight. Physical examination showed that blood pressure was $140 / 80 \mathrm{mmHg}$, pulse was 97 times per minute, body temperature was $36.3^{\circ} \mathrm{C}$, and respiratory rate was 20 times per minute. She had a clear mind, weak spirit, big and equal circles of the bilateral pupils, sensitivity to light reflection, a ruddy face, clean external auditory canals, and no deformities. The bilateral thorax was symmetrical, and breath sound was clear. The muscular tension of limbs was normal, the bilateral tendons were symmetrical, the neck strong was negative, gram sign was negative, brucelli sign was negative, and pathology sign was negative. This study was approved by the ethics committee of Renmin Hospital, Wuhan University. We obtained written, informed consent from the patient.

Routine blood examination on the first day after admission revealed the following: leukocyte, $3.45 \times 10^{9}$ (normal range, $3.5 \times 10^{9}-9.5 \times 10^{9}$ ); neutrophil, $80.70 \%$ (normal range, 50-70\%); lymphocyte, $11.60 \%$ (normal range, $20-40 \%)$.

Routine urine test was normal. Blood glucose, electrolyte, and lipid of liver function and kidney function showed that triglyceride was $3.4 \mathrm{mmol} / \mathrm{L}$. The pathogen before operation was normal. C-reactive protein (CRP) was $16.78 \mathrm{mg} / \mathrm{L}$, serum amyloid A (SAA) was $18.62 \mathrm{mg} / \mathrm{L}$, procalcitonin (PCT) was negative, HSV-1,2 DNA was negative, seven respiratory antigens were all negative, and the myocardial infarction index was normal.

Results of lumbar puncture were as follows: pressure, $125 \mathrm{mmH}_{2} \mathrm{O}$; white blood cell count, $2 \times 10^{6} / \mathrm{L}$; lymphocyte, 0.6; neutrophil, 0.07; sugar, $3.92 \mathrm{mmol} / \mathrm{L}$ (instant blood glucose, $5.3 \mathrm{mmol} / \mathrm{L}$ ), protein $0.29 \mathrm{mmol} / \mathrm{L}$, acid-fast ink-staining, negative. No bacteria, fungi, and acid-fast bacilli were found in cerebrospinal fluid (CSF) culture, and TORCH 10 was negative.

No obvious abnormality was found in the pure tone threshold, electronystagmography, or on brain magnetic resonance imaging (MRI). CT angiography (CTA) of the head and neck was normal. On the second day after admission, chest CT showed that the density of the left upper lobe and the back of the lower lobe was increased in nodular and cord shape, especially in the extrapulmonary zone. On the ninth day after admission, chest CT showed that the abnormal lesions had disappeared (Figures 1,2).

\section{Nucleic acid detection}

The 2019-nCoV (orf1 ab/N gene) nucleic acid detection Kit (double-fluorescent PCR, Shanghai Berger Medical Technology Co., Ltd., Product No.: SJ-HX-226-1,2) was used for the detection. The operation was carried out according to following the instructions of the manufacturer: First, prepare the reagent (reagent preparation area) and place each component of the kit in a $4{ }^{\circ} \mathrm{C}$ environment to avoid light melting, and thoroughly mix the reagent and sample, then centrifuge them immediately. Calculate the number of parts of the reagent $\mathrm{N}$, prepare the reaction system mix, add it into a suitable volume of centrifuge tube, mix it well, centrifuge it immediately, repack it into the PCR 

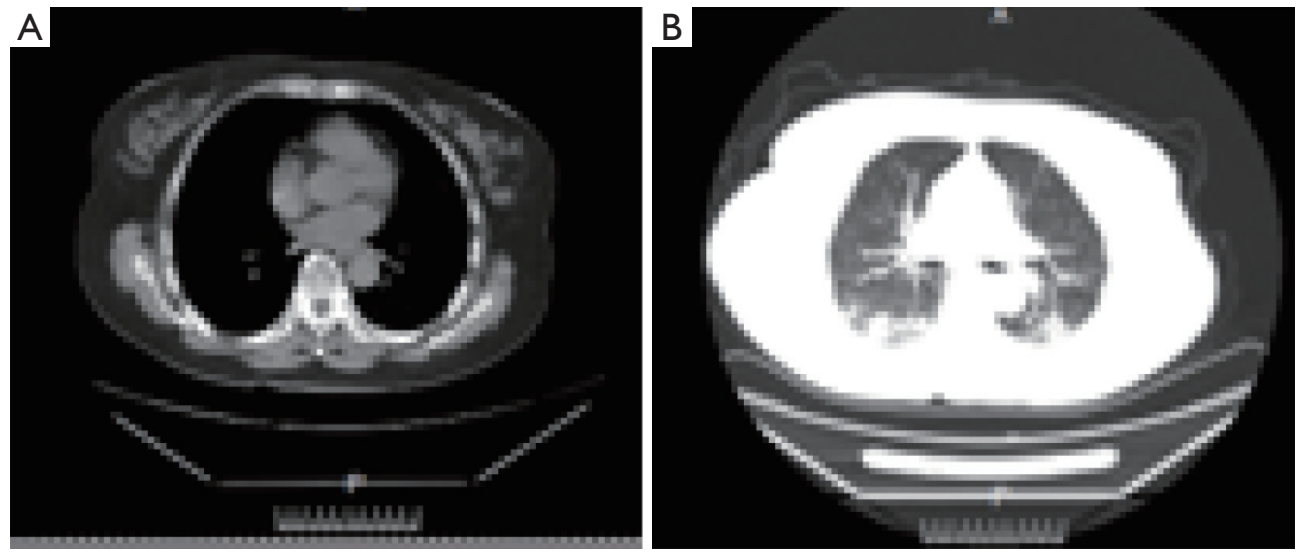

Figure 1 Chest imaging of the patient before treatment by computed tomography (CT) on the second day after admission. (A) Bone window and (B) lung window.
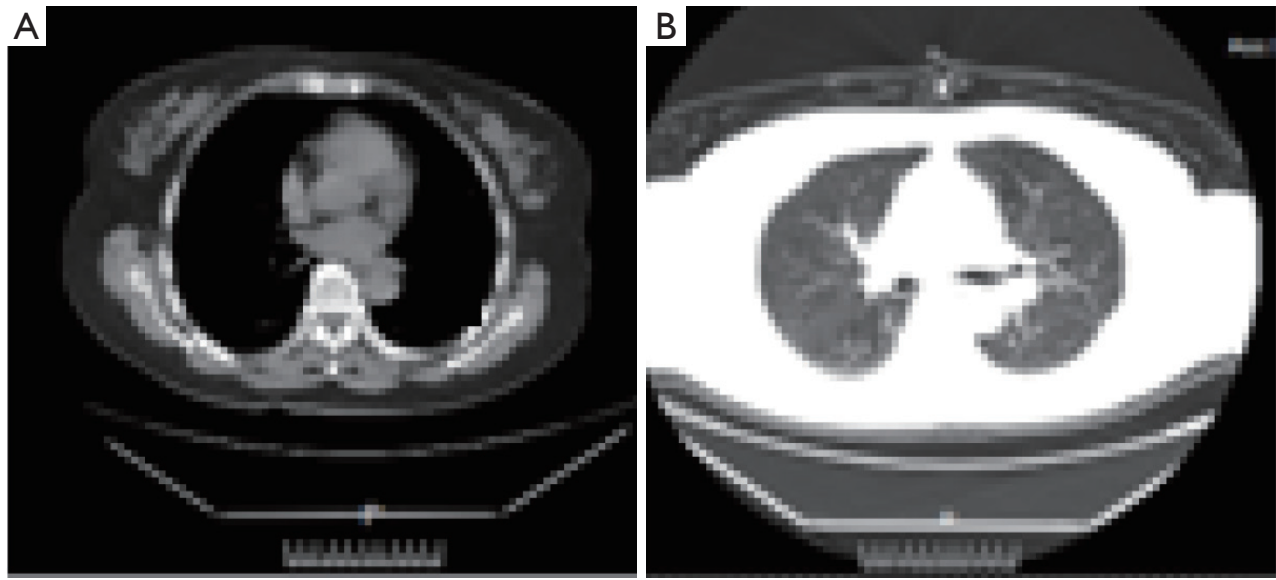

Figure 2 Chest imaging of the patient after treatment by computed tomography (CT) on the ninth day after admission. (A) Bone window and (B) lung window.

reaction tube/plate according to $20 \mu \mathrm{L}$, and transfer it to the sample processing area. After this, sample processing can be conducted as follows (sample processing area): first added the nucleic acid extraction and sample, then take the amplification test (nucleic acid amplification area). Next analysis the result in the whole experiment with control. At last, adjust the different targets to the baseline threshold according to the corresponding negative sample. On the fifth day after admission, the tests were positive for $2019-\mathrm{nCoV}$ RNA (Figure 3), and the 2019-ncov diagnosis was confirmed.

\section{Treatment and follow-up}

On the first day after admission, aspirin $0.1 \mathrm{~g}$ was given orally once a day according to acute cerebrovascular disease, lipitor $20 \mathrm{mg}$ was given orally once a day, and betahistine $6 \mathrm{mg}$ was given orally 3 times a day. An injection of $10 \mathrm{mg}$ danshenchuandomazine, a drug for improving circulation, was given once a day. The second day after admission, $2.0 \mathrm{~g}$ of ceftezole sodium for injection was given twice a day.

On the fifth day, $75 \mathrm{mg}$ of oseltamivir phosphate capsule was given twice a day, abidol $0.2 \mathrm{~g}$ was given orally 3 times a day, and baifule $0.4 \mathrm{~g}$ was given once a day. Later, the patient was transferred to a closed ward for isolation for 14 days. One month after discharge, the patient had normal dizziness and no obvious abnormality was found in the follow-up. The body temperature remained throughout the hospital admission period. The respiratory symptoms 
A
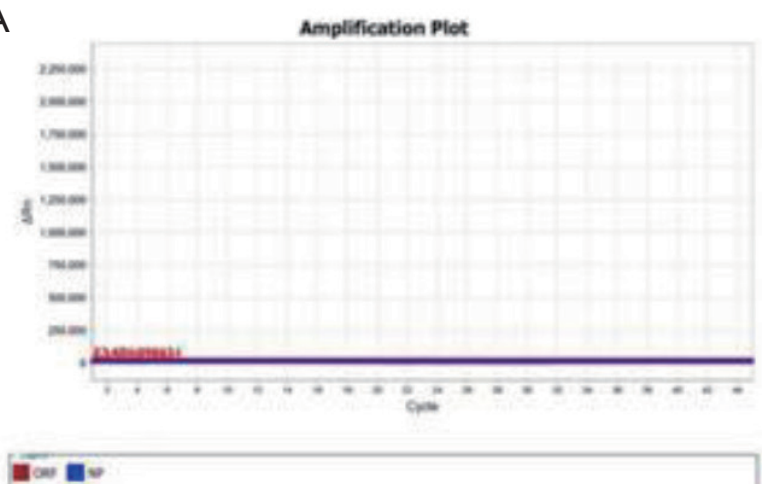

B

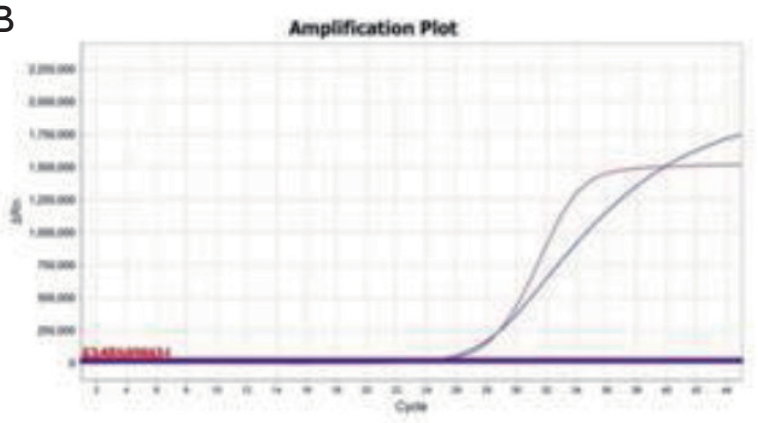

$\mathbf{0} \mathbf{0}$

Figure 3 The results of nucleic acid detection. (A) Results are from the fifth day after admission. The amplification curve was S-type and the $\mathrm{Ct}$ value $\leq 35$, which indicated a positive result. (B) Results are from the twelfth day after admission. The amplification curve was not detected and the Ct value $\geq 38$, which indicated a negative result.

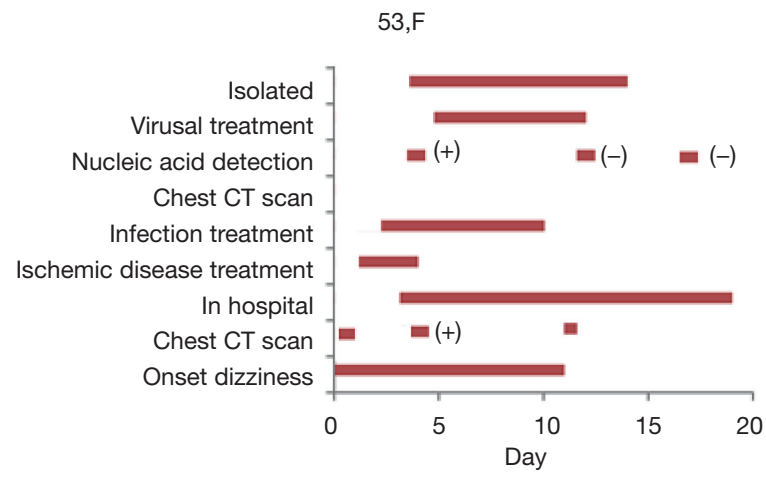

Figure 4 Timeline of the patient showing the historical and current information from this episode of care.

disappeared, the re-examination of pulmonary imaging showed that the inflammation was absorbed, and the detection of respiratory pathogenic nucleic acid was twice found to be negative. There were no symptoms of intracranial infection such as headache and fever during the course of the disease and for 2 weeks after (Figure 4).

\section{Discussion}

The number of coronavirus cases has been identified, and the number has increased day by day, with the corresponding increase in the number of coronavirus genomes, which have been sequenced (14). This increase was partly due to the discovery of the SARS coronavirus, which led to a global outbreak of pneumonia in 2003, affecting about 30 countries and killing about 800 people (8).
Before 2003, there were only 2 known human coronaviruses; $\mathrm{HCoV}-229 \mathrm{e}$ and $\mathrm{HCoV}$-oc43 were found the 1960s $(15,16)$, and in 2003, the SARS coronavirus was found. HCoVNL63 and HCoV-HKU1 may also infect humans (17-19). Although $2019-\mathrm{nCoV}$ is similar to some respiratory diseases detected in bats, it is distinct from SARS CoV and MERS $\mathrm{CoV}$ (19). Here we report the first case of a new type of pneumonia that starts with the nervous system alone.

Fever occurs most commonly after infection, and may be accompanied by fatigue, cough, chest tightness, and dyspnea. Some patients have mild onset symptoms but no fever. Most of the patients developed symptoms 1 week after infection $(3,19)$. Severe cases can progress to acute respiratory distress syndrome, septic shock, metabolic acidosis, and coagulation dysfunction, which are difficult to correct $(3,19)$. Clinical symptoms showed acute multiple organ respiratory distress syndrome (MODS), similar to the description of severe influenza cases and SARS (19-21).

According to the existing clinical data and experience, those who meet the following conditions are all highrisk suspected cases (3): within 14 days before the onset of the disease, there is a history of travel or residence in Wuhan city; within 14 days before the onset of the disease, they have been exposed to patients with fever from Wuhan accompanied by respiratory symptoms, or they have clustering disease; the patients have the imaging characteristics of pneumonia while they are hot; the total number of leukocytes in the early stage of the disease is normal or decreased, or the decrease of lymphocyte count can be judged as a suspected case (18).

At the same time, most of the patients are mild, with 
the remainder being critically ill or even dead. In this case, the early manifestations of chest CT were multiple small spot films and interstitial changes, especially in the extrapulmonary zone, which is in line with the reported literature (22). Some people may develop multiple ground-glass shadows and infiltrative shadows in both lungs (22), but our case did not deteriorate to this state. Blood routine examination in the early stage of the disease can show if the total number of peripheral blood leukocytes is normal or has decreased. In most patients, C-reactive protein and erythrocyte sedimentation rate (ESR) is increased, and procalcitonin is normal. The nucleic acid 2019-nCoV virus test showed doublepositive, which further supports the 2019-nCoV diagnosis. To compare with the literature, the hematological changes of SARS patients were reported to be found in lymphocytopenia, neutropenia, and late thrombocytopenia, and these patients exhibited abnormal variables of hematology. Lymphocytopenia was the most severe common finding in the 157-patient cohort of SARS (8). In these patients, organ or reactive hemophagocytic syndrome with lymphopenia and no marrow failure features were found after death. They also had progressive damage to renal function, which was similar to those SARS patients who might have had directly infected renal tissue. Overall, it can be seen that the recognition of coronavirus is a longterm process. The patient we reported is mild and has a good prognosis. This also suggests that the pathogenicity of 2019-nCoV may be slightly weak.

Though this is a single case, with the further spread and greater understanding of the disease, there will likely be similar reports in the future. The $2019-\mathrm{nCoV}$ epidemic is continuing, and the number of infections and deaths are increasing dramatically. In Wuhan, we have taken active measures to prevent and control the spread of infection. It is of considerable significance to identify the infected early and cut off the source of infection. To control and prevent the spread of 2019-nCoV, it is essential to use key epidemiological surveys that clarify transmission patterns, recurrence intervals, and the clinical symptom spectrum caused by infection, so that more informed preventive strategies can be refined.

\section{Acknowledgments}

We would like to thank Qun Wang (Beijing Tiantan Hospital, Capital Medical University, Beijing, China) for editing the manuscript for English grammar.
Funding: Grants were given to Dr. Zhaohong Hong by the Wu Jieping Medical Foundation (number: 320.6750.1909223), and to Dr. Zhaohui Zhang by The National Natural Science Foundation (number: 81671051).

\section{Footnote}

Conflicts of Interest: All authors have completed the ICMJE uniform disclosure form (available at http://dx.doi. org/10.21037/atm.2020.03.89). The authors report grants from Wu Jieping Medical Foundation, and The National Natural Science Foundation, outside the submitted work.

Ethical Statement: The authors are accountable for all aspects of the work in ensuring that questions related to the accuracy or integrity of any part of the work are appropriately investigated and resolved. Written informed consent was obtained from the patient for publication of this manuscript and any accompanying images.

Open Access Statement: This is an Open Access article distributed in accordance with the Creative Commons Attribution-NonCommercial-NoDerivs 4.0 International License (CC BY-NC-ND 4.0), which permits the noncommercial replication and distribution of the article with the strict proviso that no changes or edits are made and the original work is properly cited (including links to both the formal publication through the relevant DOI and the license). See: https://creativecommons.org/licenses/by-nc-nd/4.0/.

\section{References}

1. Bogoch II, Watts A, Thomas-Bachli A, et al. Potential for global spread of a novel coronavirus from China. J Travel Med 2020. [Epub ahead of print].

2. Gao GF. From "A"IV to "Z"IKV: attacks from emerging and re-emerging pathogens. Cell 2018;172:1157-9.

3. Zhu N, Zhang D, Wang W, et al. A Novel Coronavirus from Patients with Pneumonia in China, 2019. N Engl J Med 2020;382:727-33.

4. Behzadi MA, Leyva-Grado VH. Overview of Current Therapeutics and Novel Candidates Against Influenza, Respiratory Syncytial Virus, and Middle East Respiratory Syndrome Coronavirus Infections. Front Microbiol 2019;10:1327.

5. Masters PS, Perlman S. Coronaviridae. In: Knipe DM, Howley PM. editors. Fields virology. 6th ed. Lippincott Williams \&Wilkins, 2013:825-58. 
6. Su S, Wong G, Shi W, et al. Epidemiology, genetic recombination, and pathogenesis of coronaviruses. Trends Microbiol 2016;24:490-502.

7. Cui J, Li F, Shi ZL. Origin and evolution of pathogenic coronaviruses. Nat Rev Microbiol 2019;17:181-92.

8. Zhong NS, Zheng BJ, Li YM, et al. Epidemiology and cause of severe acute respiratory syndrome (SARS) in Guangdong, People's Republic of China, in February, 2003. Lancet 2003;362:1353-8.

9. Ksiazek TG, Erdman D, Goldsmith CS, et al. A novel coronavirus associated with severe acute respiratory syndrome. N Engl J Med 2003;348:1953-66.

10. Drosten C, Günther S, Preiser W, et al. Identification of a novel coronavirus in patients with severe acute respiratory syndrome. N Engl J Med 2003;348:1967-76.

11. Zaki AM, van Boheemen S, Bestebroer TM, et al. Isolation of a novel coronavirus from a man with pneumonia in Saudi Arabia. N Engl J Med 2012;367:1814-20.

12. Wong G, Liu W, Liu Y, et al. MERS, SARS, and Ebola: the role of super-spreaders in infectious disease. Cell Host Microbe 2015;18:398-401.

13. Riley DS, Barber MS, Kienle GS, et al. CARE 2013 Explanations and Elaborations: Reporting Guidelines for Case Reports. J Clin Epidemiol 2017;89:218-35.

14. Chen Y, Liu Q, Guo D. Emerging coronaviruses: genome

Cite this article as: Kong Z, Wang J, Li T, Zhang Z, Jian J. 2019 novel coronavirus pneumonia with onset of dizziness: a case report. Ann Transl Med 2020;8(7):506. doi: 10.21037/ atm.2020.03.89 structure, replication, and pathogenesis. J Med Virol 2020;92:418-23.

15. Jonsdottir HR, Dijkman R. Coronaviruses and the human airway: a universal system for virus-host interaction studies. Virol J 2016;13:24.

16. Palacios G, Druce J, Du L, et al. A new arenavirus in a cluster of fatal transplant associated diseases. N Engl J Med 2008;358:991-8.

17. Tan WJ, Zhao X, Ma XJ, et al. A novel coronavirus genome identified in a cluster of pneumonia cases-Wuhan, China 2019-2020. China CDC Weekly 2020;2:61-2.

18. Armstrong GL, MacCannell DR, Taylor J, et al. Pathogen genomics in public health. N Engl J Med 2019;381:2569-80.

19. Huang C, Wang Y, Li X, et al. Clinical features of patients infected with 2019 novel coronavirus in Wuhan, China. Lancet 2020;395:497-506.

20. Kaw GJ, Tan DY, Leo YS, et al. Chest radiographic findings of a case of severe acute respiratory syndrome (SARS) in Singapore. Singapore Med J 2003;44:201-4.

21. Fraaij PL, Heikkinen T. Seasonal influenza: the burden of disease in children. Vaccine 2011;29:7524-8.

22. Lei J, Li J, Li X, et al. CT Imaging of the 2019 Novel Coronavirus (2019-nCoV) Pneumonia. Radiology 2020. [Epub ahead of print]. 